\title{
Effect of mobility devices on orientation sensors that contain
}

\section{magnetometers}

\author{
Cynthia Kendell, BSc; ${ }^{*}$ Edward D. Lemaire, PhD \\ The Ottawa Hospital Rehabilitation Centre, The Ottawa Hospital, Ottawa, Ontario, Canada
}

\begin{abstract}
Orientation sensors containing magnetometers use the earth's magnetic field as a reference. Ferromagnetic objects may distort this magnetic field, leading to inaccurate orientation output. We explored the viability of these orientation sensors for motion analysis in an assistive mobility device rehabilitative setting. We attached two MTx orientation sensors (XSens; Enschade, the Netherlands), connected to the XBus Master data collection unit (XSens), to a plastic frame such that the relative angle between sensors was constant. We then moved a series of mobility devices in proximity to the plastic frame: two knee-ankle-foot orthoses (aluminum, stainless steel), one ankle-foot orthosis, two transtibial prostheses (exoskeletal, endoskeletal), two walkers (standard, Challenger Low Wide [Evolution Technologies; Port Coquitlam, Canada]), and two wheelchairs (Tango [OrthoFab; Quebec City, Canada], GTi [Quickie; Phoenix, Arizona]). For each mobility device, we calculated the average difference in relative angle between the baseline and peak angles for each of five trials. Errors ranged from less than 0.10 to 35.29 degrees, depending on the mobility device and frame positioning near the device. This demonstrated the large errors that can occur when magnetometerbased orientation sensors with mobility devices are used. While strategic orientation sensor placement on some mobility devices can minimize these errors to an acceptable level, testing protocols should be implemented to verify orientation sensor accuracy for these applications.
\end{abstract}

Key words: accelerometers, biomechanics, gyroscopes, inertial sensors, kinematics, magnetometers, mobility device, motion analysis, orientation sensor, rehabilitation.

\section{INTRODUCTION}

Accelerometers and similar inertial devices have a long history of use in human motion analysis. Recent technological advances in microelectromechanical systems have lead to new accelerometer, gyroscope, and magnetometer devices that can be packaged into a relatively small unit $(4 \times 5 \times 2 \mathrm{~cm})$ [1]. These sensors could be an effective alternative to popular optoelectric motion analysis systems since their small profile permits use under clothing and, with appropriate data-logging, can be used outside laboratory settings. This technology could prove especially useful for monitoring the progress of rehabilitation patients and assessing their mobility at home and in the community.

While these orientation sensors have various limitations when used separately, combining accelerometergyroscope-magnetometer sensor data can improve on individual orientation sensor deficiencies. In the absence of acceleration, accelerometers can be used to measure the angle of the orientation sensor in respect to gravity [2]; however, accelerometers cannot provide information

Abbreviations: 3-D $=$ three-dimensional, $\mathrm{KAFO}=$ knee-anklefoot orthosis, RMS = root mean square.

*Address all correspondence to Cynthia Kendell, BSc; The Ottawa Hospital Rehabilitation Centre, 505 Smyth Road, Ottawa, ON K1H 8M2 Canada; 613-737-8899, ext 75326; fax: 613-737-4260. Email: clkendell@yahoo.ca

DOI:10.1682/JRRD.2008.09.0132 
about rotation around the vertical axis. Orientation information from accelerometers can be supplemented with angular velocity data from gyroscopes. Unfortunately, gyroscopes are not without issue-a small error in the measurement of angular velocity can result in increasingly large errors in estimated orientation (i.e., integration errors) [2]. Magnetometers use the earth's magnetic field as a reference and can provide information on rotation about the vertical axis and correct for gyroscope integration errors [3]. Unfortunately, using the earth's magnetic field as a reference can result in sensor output errors. Ferromagnetic materials will disturb the earth's magnetic field measured by the magnetometers. As with a compass, magnetic disturbances affect output related to rotation about the vertical axis [3-4].

Several studies have explored the use of combinations of orientation sensors for motion analysis applications. Nene et al. used uniaxial accelerometers and a gyroscope to calculate absolute angle, angular velocity, and moments about the knee [5]. In ergonomics, Veltink et al. used triaxial accelerometers to measure pelvic angular acceleration during crate stacking [6]. Similarly, Plamondon et al. created a hybrid system comprised of gyroscopes, accelerometers, magnetometers, and a pentiometer to measure three-dimensional (3-D) kinematics during bending tasks [7].

Luinge et al. used accelerometer and gyroscope signals to examine upper-limb movement during a variety of daily-living tasks (i.e., combing hair, pouring a glass of water) [2,4]. In other applications, Roetenberg et al. combined accelerometers, gyroscopes, and magnetometers to measure displacement from a known source [8] and Pfau et al. used commercial orientation sensors (XSens; Enschede, the Netherlands) to measure equestrian trunk movement during treadmill locomotion [9].

With the exception of studies by Luinge et al., the range of error observed with the use of inertial sensors has been reported as acceptable [2,4]. In cases where inertial sensors have been validated against other optoelectric methods, the two systems have produced comparable results [6-10], with the root mean square (RMS) error varying from less than $2^{\circ}[10]$ to approximately $10^{\circ}$ [7]. In each of these studies, a magnetometer was incorporated into the inertial sensor. Unsurprisingly, the authors of the articles consistently cited magnetic interference as a possible source of error.

We evaluated commercially available orientation sensors that incorporate accelerometers, gyroscopes, and magnetometers (XSens). With these orientation sensors, two types of magnetic interference may be problematic: deterministic and nondeterministic [11]. Deterministic interference occurs when the orientation sensors are affixed to an object that contains ferromagnetic materials such that the relationship between the orientation sensors and the object remains constant so we can predict the error in the magnetic field. Nondeterministic disturbance occurs when a ferromagnetic object moves independently with respect to the orientation sensors. The MT Software Development Kit (XSens) offers a calibration procedure meant to eliminate deterministic disturbance, but nondeterministic disturbance cannot be compensated for [11].

When we examine motion in a rehabilitation environment, patients often use assistive mobility devices. Unfortunately, nondeterministic interference becomes an issue in this setting because the relationship between body segments and the mobility device is not fixed. For example, if the orientation sensors were affixed to the upper limbs of a wheelchair user, the relationship between the metal in the wheelchair and the sensors would not remain constant. The same problem occurs for orientation sensors affixed to the lower limbs of an individual using a walker. In the case of orthoses and prostheses, in which the orientation sensors would likely be attached to the mobility device, the metal in the device could affect orientation sensors on the contralateral limb.

The objectives of this article are to quantify the error caused by assistive mobility devices on commercially available orientation sensors that contain magnetometers and to examine the viability of using these orientation sensors in rehabilitation research.

\section{METHODS}

We collected data by using MTx orientation sensors (XSens). We connected these orientation sensors to an XBus Master data collection unit (XSens) in series. Each MTx orientation sensor is comprised of three orthogonally mounted gyroscopes, accelerometers, and magnetometers housed in a plastic casing. An orientation sensor fusion algorithm calculates absolute 3-D orientation by combining output from all three components [1]. The algorithm uses gravity and magnetic north as a reference to correct for gyroscope integration drift. When using orientation output mode, XSens documentation reports that 
the angular dynamic accuracy is $2^{\circ} \mathrm{RMS}$, depending on the motion type [1].

To test for the effect of assistive mobility devices on sensor-fused orientation output, we used the following devices: exoskeletal transtibial prosthesis, endoskeletal transtibial prosthesis, knee-ankle-foot orthosis (KAFO) with aluminum uprights, KAFO with stainless steel uprights, ankle-foot orthosis, Challenger Low Wide walker (Evolution Technologies; Port Coquitlam, Canada), standard walker, Tango wheelchair (OrthoFab; Quebec City, Canada), and GTi wheelchair (Quickie; Phoenix, Arizona).

We affixed two MTx orientation sensors to a Plexiglass " $\mathrm{L}$ " so that the relative angles between the orientation sensors remained fixed. We mounted the L securely to the side of a plastic box. At their closest point, the orientation sensors were $21.5 \mathrm{~cm}$ apart (Figure 1).

To establish baseline relative angles, we collected $10 \mathrm{~s}$ of MTx orientation sensor data with the experimental set up in a stationary position. After $10 \mathrm{~s}$, we moved a selected assistive mobility device within $3 \mathrm{~cm}$ of the orientation sensor(s) and then we removed it. We repeated the mobility device movement step five times for each

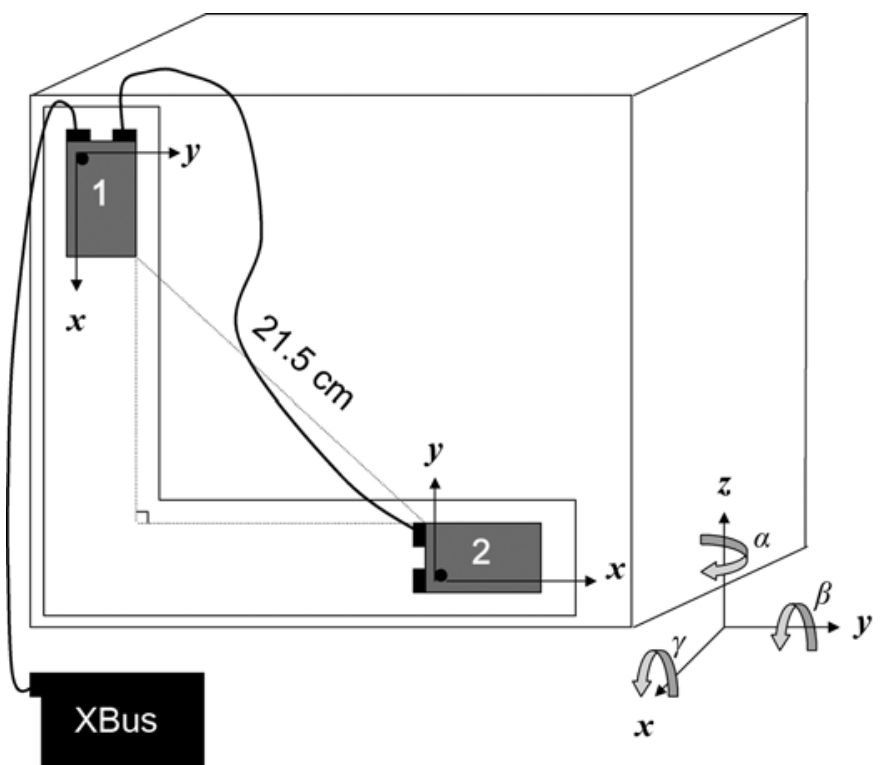

Figure 1.

Experimental setup and coordinate system. Rotations refer to changes in relative angle when expressing sensor 2 (2) in respect to sensor 1 (1). XBus $=$ XBus Master data collection unit (XSens; Enschade, the Netherlands), $\alpha=$ rotation about $z$-axis, $\beta=$ rotation about $y$-axis, $\gamma=$ rotation about $x$-axis. device. The next subsections describe the protocol for each type of mobility device.

\section{Orthoses and Prostheses}

We positioned the plastic box with the MTx orientation sensors affixed on a cardboard box in an area at least $1 \mathrm{~m}$ from ferromagnetic materials. To test the effect of metal in different parts of each mobility device, we moved the shank of the orthosis or prosthesis near the top orientation sensor (sensor 1) and then we removed it (repeated five times). We completed the same procedure for the thigh, footplate, and joint components for each orthosis and prosthesis.

\section{Walkers}

We placed the plastic box on a plastic platform on the ground to minimize possible effects of metal in the floor. The distance from the ground to the closest sensor was $19 \mathrm{~cm}$. With the box stationary, we rolled each walker to the box and then we removed it (repeated five times).

\section{Wheelchairs}

We suspended the plastic box from the ceiling by ropes approximately $3 \mathrm{~cm}$ above the seat of each wheelchair. This rigging kept the box stationary during testing. We wheeled each wheelchair to the box so that the box was suspended within the area of the wheelchair seat, and then we pulled the wheelchair away (repeated five times).

\section{DATA ANALYSIS}

We saved data as directional cosine matrices from the two MTx orientation sensors as text files and imported them into Excel (Microsoft Corp; Redmond, Washington) for processing and analysis. For all trials, we excluded the first $5 \mathrm{~s}$ of data to ensure that the MTx orientation sensors had achieved a steady state. We calculated the relative Euler angles between the two orientation sensors by expressing the relative orientation of sensor 2 with respect to sensor 1, using equations provided by XSens [1]. We graphically plotted Euler angles for five trials for each mobility device.

For trials in which magnetic field disturbance was evident about at least one axis (i.e., observable relative angle fluctuation), we calculated baseline angles and extracted peak angles for each trial and for each mobility device. We calculated the baseline angles by averaging 
the relative angles between 5 and $7 \mathrm{~s}$ of data collection (i.e., before we introduced the assistive mobility device). We averaged peak angles for each mobility device over five trials. We calculated the difference between baseline and average peak angle for each mobility device.

For trials in which no magnetic disturbance was evident (i.e., five peaks were not present), we calculated the difference between the baseline relative angle and average relative angle. We calculated the average relative angle by averaging relative angles over the duration of data collection, excluding the first $10 \mathrm{~s}$ of each trial.

\section{RESULTS}

Since the MTx orientation sensors remained in a fixed position during each test, we considered any fluctuation in relative angle from the baseline an error. Figure 2 contains the data for trials in which we observed magnetic disturbance about at least one of the axes. We extracted peak values from the same range of data points for each axis. As illustrated in Figure 3, when magnetic disturbances occurred, the relative angle about the affected axis changed and formed five identifiable peaks (one peak per trial). Figure 4 contains the data for trials in which we observed no disturbance.

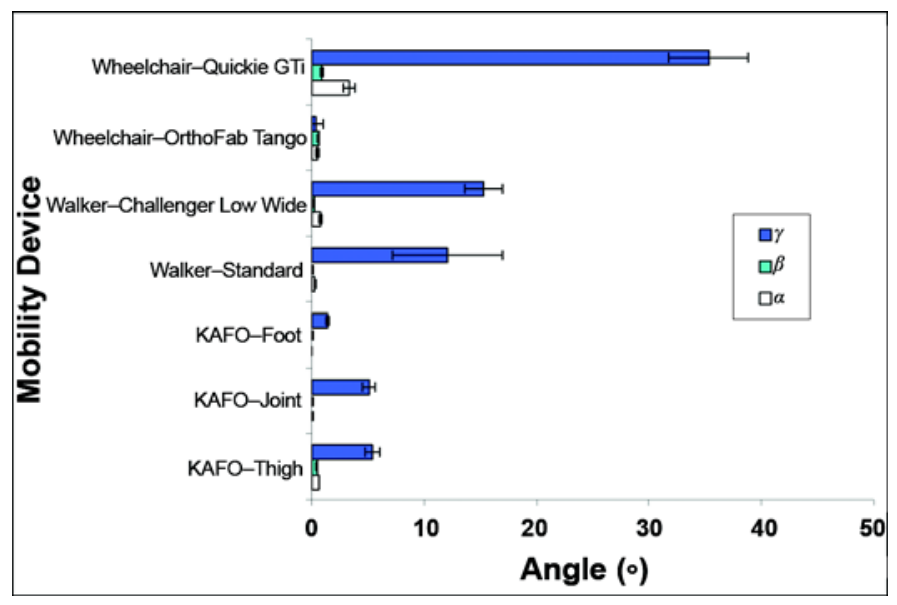

Figure 2.

Observed disturbance. Average difference \pm standard deviation between peak and baseline relative angles for each assistive mobility device displaying magnetic disturbance about at least one axis. Rotations refer to changes in relative angle when expressing sensor 2 with respect to sensor 1 . KAFO $=$ knee-ankle-foot orthosis with stainless steel uprights, $\alpha=$ rotation about $z$-axis, $\beta=$ rotation about $y$-axis, $\gamma=$ rotation about $x$-axis.

\section{DISCUSSION}

We investigated the usefulness of commercially available orientation sensors that incorporate a magnetometer for kinematic analysis in cases where the subject uses an mobility device. We exposed the orientation sensors to a variety of mobility devices and examined whether sensor output was affected and to what magnitude.

Since rapid acceleration can produce erroneous inclination values for accelerometers, such errors could affect angular output. We controlled for rapid acceleration errors in this study since the orientation sensors remained stationary throughout each test. Therefore, we attributed errors (changes in relative angle) to disturbances in the earth's magnetic field that affected magnetometer output.

The effect of each assistive mobility device on the MTx orientation sensor output varied greatly between devices. We found the greatest change in relative angle

(a)

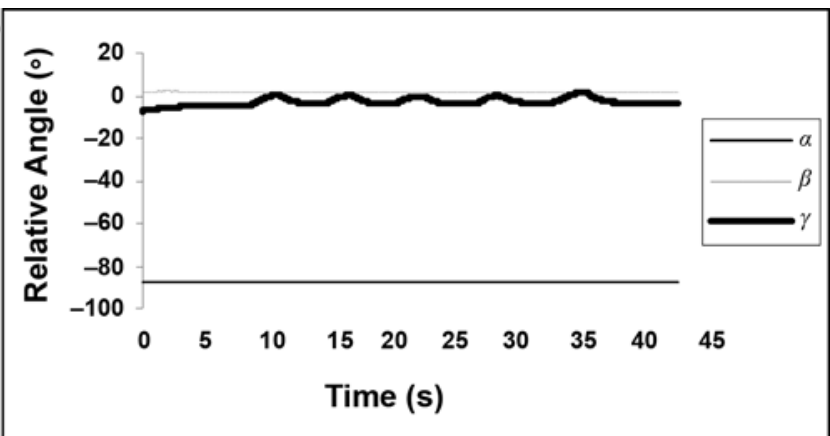

(b)

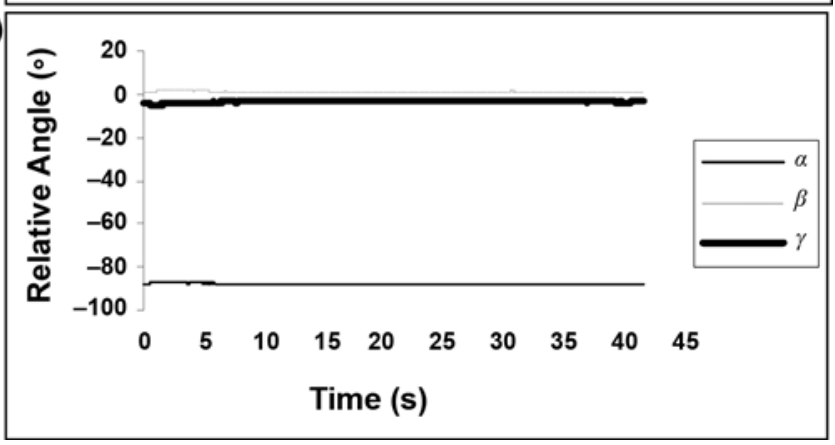

Figure 3.

Relative angles observed when we introduced (a) joint component of knee-ankle-foot orthosis with stainless steel uprights and (b) foot of endoskeletal prosthesis to proximity of one orientation sensor (sensor 1). Rotations refer to changes in relative angle when expressing sensor 2 with respect to sensor 1. $\alpha=$ rotation about $z$-axis, $\beta=$ rotation about $y$-axis, $\gamma=$ rotation about $x$-axis. 


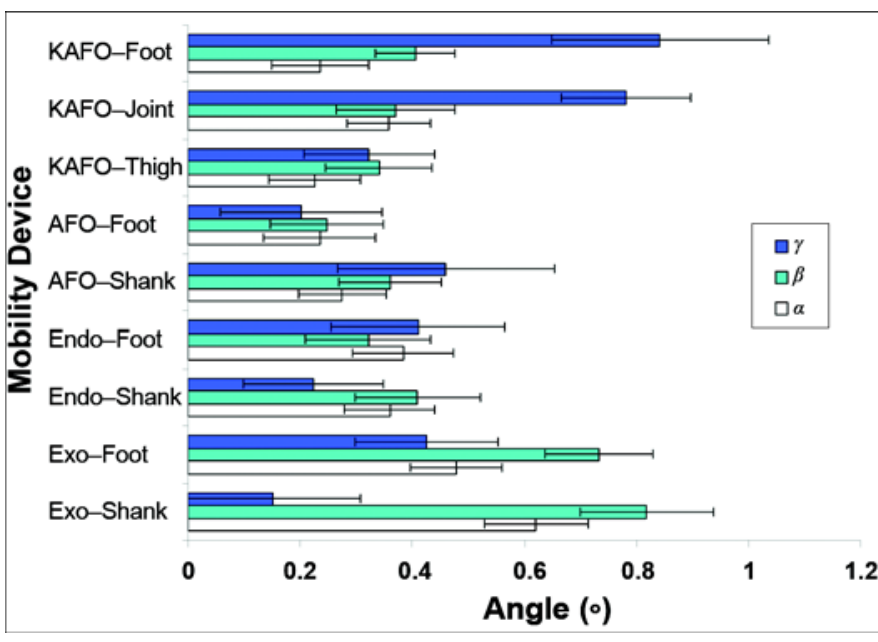

Figure 4.

No observed disturbance. Average difference \pm standard deviation between baseline relative angle (5-7 s) and relative angles from $10 \mathrm{~s}$ on. Rotations refer to changes in relative angle when expressing sensor 2 with respect to sensor 1 . Endo = endoskeletal prosthesis, Exo = exoskeletal prosthesis, KAFO = knee-ankle-foot orthosis with aluminum uprights, $\alpha=$ rotation about $z$-axis, $\beta=$ rotation about $y$-axis, $\gamma=$ rotation about $x$-axis.

with the GTi wheelchair with an average error of $35.29^{\circ}$. We also found substantial relative angle differences for different parts of the same mobility device. For example, the range of error for the KAFO with stainless steel uprights varied from $5.40^{\circ}$ for the thigh component to $1.43^{\circ}$ for the foot component. The difference in the magnitude of error relates to the amount of metal present in each component of the mobility device. For the majority of trials, we found no effect on the relative angle in all planes, indicating that the mobility device did not distort the earth's magnetic field. Figure $\mathbf{4}$ shows that we found very little relative angle fluctuation for these trials, as evidenced by the standard deviation.

This article supports the idea that magnetic interference only affects heading, or rotation about the vertical axis. For our study, we defined the vertical axis as $x$ since the relative angles are expressed as the orientation of sensor 2 with respect to sensor 1 . Results indicated that when we observed interference, rotation about $x(\gamma)$ was affected most frequently and to the greatest magnitude.

The MTx orientation sensors produced reasonably accurate data before being introduced to the mobility devices. The baseline relative angles should have reflected rotations of $0^{\circ}, 0^{\circ}$, and $90^{\circ}$ about $x, y$, and $z$, respectively. Baseline relative angles ranged from $-3^{\circ}$ to $-6^{\circ}$ for $x, 1^{\circ}$ to $2^{\circ}$ for $y$, and $87^{\circ}$ to $88^{\circ}$ for $z$. This deviation from expected values may be due to initial positioning of the MTx orientation sensors or to using the external casing to align the MTx orientation sensors. XSens notes that the internal sensors are not exactly aligned to the casing and may be offset by up to $3^{\circ}[1]$.

\section{CONCLUSIONS}

Orientation sensors containing magnetometers may not produce reliable data when used to analyze the motion of individuals using assistive mobility devices, depending on the type and amount of metal used to fabricate the device. For this reason, we recommend that preliminary testing before motion analysis be used to determine whether the metals used in the mobility device illicit a disturbance in the orientation data. When orientation sensors are mounted to a mobility device, such tests can also be used to determine optimal placement. Future research should investigate optimal orientation sensor placement and possible modification of the calibration process outlined by XSens.

\section{ACKNOWLEDGMENTS}

\section{Author Contributions:}

Study concept and design: C. Kendell, E. D. Lemaire. Acquisition of data: C. Kendell.

Analysis and interpretation of data: C. Kendell, E. D. Lemaire. Drafting of manuscript: C. Kendell.

Critical revision of manuscript for important intellectual content: E. D. Lemaire.

Statistical analysis: C. Kendell, E. D. Lemaire.

Obtained funding: E. D. Lemaire.

Administrative, technical, or material support: E. D. Lemaire.

Financial Disclosures: The authors have declared that no competing interests exist.

Funding/Support: This material was based on work supported by the Atlantic Innovation Fund (grant 181909).

Additional Contributions: We would like to thank Terris Yakimovich and the Ottawa Hospital Rehabilitation Centre for technical and facility support for this project. We would also like to acknowledge the Atlantic Innovation Fund for financial assistance related to purchasing the XSens system and research assistant support.

\section{REFERENCES}

1. MTi and MTx user manual and technical documentation. Enschede (the Netherlands): XSens; 2006. 
2. Luinge HJ, Veltink PH. Measuring orientation of human body segments using miniature gyroscopes and accelerometers. Med Biol Eng Comput. 2005;43(2):273-82.

[PMID: 15865139]

DOI:10.1007/BF02345966

3. Roetenberg D, Luinge HJ, Baten CT, Veltink PH. Compensation of the magnetic disturbances improves inertial and magnetic sensing of human body segment orientation. IEEE Trans Neural Syst Rehabil Eng. 2005;13(3):395-405. [PMID: 16200762] DOI:10.1109/TNSRE.2005.847353

4. Luinge HJ, Veltink PH, Baten CT. Ambulatory measurement of arm orientation. J Biomech. 2007;40(1):78-85. [PMID: 16455089]

DOI:10.1016/j.jbiomech.2005.11.011

5. Nene A, Mayagoitia R, Veltink P. Assessment of rectus femoris function during initial swing phase. Gait Posture. 1999;9(1):1-9. [PMID: 10575064]

DOI:10.1016/S0966-6362(98)00042-3

6. Veltink PH, Luinge HJ, Kooi BJ, Baten CT, Slycke P, Olthuis W, Bergveld P. The artificial vestibular system-Design of a tri-axial inertial sensor system and its application to the study of human movement. Symposium of the International Society for Posture and Gait Research: Control of Posture and Gait; 2001; Maastricht, the Netherlands.
7. Plamondon A, Delisile A, Larue C, Brouillette D, McFadden D, Desjardins P, Larivière C. Evaluation of a hybrid system for three-dimensional measurement of trunk posture in motion. Appl Ergon. 2007;38(6):697-712.

[PMID: 17382283]

DOI:10.1016/j.apergo.2006.12.006

8. Roetenberg D, Slycke PJ, Veltink PH. Ambulatory position and orientation tracking fusing magnetic and inertial sensing. IEEE Trans Neural Biomed Eng. 2007;54(5):883-90. [PMID: 17518285] DOI:10.1109/TBME.2006.889184

9. Pfau T, Witte TH, Wilson AM. A method for deriving displacement data during cyclical movement using an inertial sensor. J Exp Biol. 2005;208(Pt 13):2503-14.

[PMID: 15961737]

DOI:10.1242/jeb.01658

10. Picerno P, Cereatti A, Capozzo A. Inertial and magnetic motion tracking: Anatomical calibration. Gait Posture. 2006; 24:S23-24. DOI:10.1016/j.gaitpost.2006.09.040

11. Magnetic field mapper MT software add-on user manual. Enschede (the Netherlands): XSens; 2005.

Submitted for publication September 29, 2008. Accepted in revised form March 30, 2009. 